\title{
Preparation of vesicular stomatitis virus pseudotype with Chikungunya virus envelope protein
}

\author{
W. TONG ${ }^{1}$, X.-X. YIN ${ }^{1}$, B.-J. LEE 2 , Y.-G. LI ${ }^{*}$ \\ ${ }^{1}$ Department of Pathogenic Microbiology, Liaoning Medical University, Jinzhou 121000, Liaoning Province, P. R. China; \\ ${ }^{2}$ Korea Institute of Toxicology, Daejeon, Republic of Korea
}

Received October 10, 2014; accepted May 15, 2015

\begin{abstract}
Summary. - Chikungunya virus (CHIKV) is a mosquito-transmitted alphavirus that causes Chikungunya fever (CHIKF) in millions of people mainly in developing countries. CHIKF is characterized by high fever, fatigue, headache, nausea, vomiting, rash, myalgia and severe arthralgia. To date, there is no specific treatment and no licensed vaccine against CHIKV infection. In this study, we developed a safe, efficient and easy neutralization assay of CHIKV based on vesicular stomatitis virus (VSV) pseudotype with CHIKV envelope protein and the green fluorescent protein (GFP) or luciferase as reporter gene, which could be used under a reduced safety level. The VSV pseudotype can be applied to the epidemic survey by measuring the expression of GFP or luciferase activity in infected cells. This system can also be used to study the mechanisms of virus entry.
\end{abstract}

Keywords: Chikungunya virus; pseudotype; envelope protein; neutralization

\section{Introduction}

Chikungunya virus (CHIKV), an alphavirus belonging to the Togaviridae family, was first isolated from a febrile individual in Tanzania in 1952 (Strauss et al., 1986; Peters et al., 1990). CHIKV is transmitted to humans by several species of mosquitoes, with Aedes aegypti and A. albopictus being the two main vectors. Throughout the world, CHIKV has affected as many as 3-4 millions people in the Indian Ocean zone and has spread to continental Europe in 2005-2007. $\mathrm{CHIKV}$ has recently received considerable attention, and many parallels may be drawn between CHIKV diseases and the other alphaviral arthritides, such as Ross River virus (RRV) disease. The symptoms of CHIKV infection generally start 4-7 days after the bite. Acute infection lasts 1-10 days and is characterized by a painful polyarthralgia, high fever,

*Corresponding author. E-mail: lygjo@hotmail.com; phone:+86(0)416-4673251.

Abbreviations: $\mathrm{CHIKF}=$ Chikungunya fever; $\mathrm{CHIKV}=$ Chikungunya virus; $\mathrm{GFP}$ = green fluorescent protein; $\mathrm{IU}$ = infectious units; $\mathrm{NT}=$ neutralization; VSV = vesicular stomatitis virus asthenia, headache, vomiting, rash, and myalgia in most patients (Burt et al., 2001; Schwartz and Albert, 2010). However, it is difficult to diagnosis because similar symptoms can be seen in the dengue virus infection. It would be beneficial to develop a system that can be easily and conveniently used to carry out epidemic survey.

$\mathrm{CHIKV}$ is a positive-sense single-stranded RNA virus, and its genome is $\sim 11.8 \mathrm{~kb}$ long with $5^{\prime}$ cap and poly (A) tail structures (Khan et al., 2002; Solignat et al., 2009). It contains two open reading frames, one for nonstructural polyprotein that is processed into four proteins and the other for a structural polyprotein that produces the capsid and the E3, E2, 6k, and $\mathrm{E} 1$ proteins. $\mathrm{E} 2$ and $\mathrm{E} 1$ form a heterodimer that projects as an outward-facing spike on the surface of CHIKV particles and, as with other alphaviruses, the E2 protein is located at the tip of the spike, so that it is likely to become the target of neutralizing antibodies (Voss et al., 2010). As far as we know, the surface protein of enveloped viruses plays an important role in the infection of target cells as it mediates the attachment of virions to the cellular membrane. A convenient way to analyze the first steps during a viral life cycle is the use of viral pseudotypes, where the viral surface protein of 
interest is incorporated into the envelope of an unrelated virus. Since the helper virus is defective, allowing virus entry but not the release of infectious virus, pseudotyped viruses provide a safe tool to analyze viral proteins of highly pathogenic viruses under conventional biosafety conditions. Furthermore, results obtained for a surface protein of an enveloped virus are not affected by other proteins of this virus. Most pseudotype systems are based on lentiviruses or VSV, a member of the family Rhabdoviridae. The generation of replication-incompetent VSV, in which the open reading frame for the VSV-G glycoprotein is replaced by that for the green fluorescent protein (GFP), facilitates the readout of the VSV system (Takada et al., 1997; Fukushi et al., 2005). Other reporter systems depend on enzymes, such as luciferase, to demonstrate the infection of target cells (Chan et al., 2000). The latter enzyme makes the assay very sensitive, although it does not indicate the number of infected cells. Because of the high sensitivity of this pseudotype system it can even be used for viral glycoproteins that are generally not transported to the budding site of helper virus. The pseudotype system with VSV particle (the VSV $\Delta \mathrm{G}^{*}$ system, in which the VSV-G is replaced by the GFP gene) was reported previously to produce VSV pseudotype particles incorporating the envelope glycoproteins of several RNA viruses, i.e. measles virus, hantavirus, Ebola virus or hepatitis $\mathrm{C}$ virus (Chen and Okayama, 1987; Niwa et al., 1991; Takada et al., 1997; Tatsuo et al., 2000; Matsuura et al., 2001; Khan et al., 2002; Ogino et al., 2003; Fukushi et al., 2005; Schuffenecker et al., 2006; Sourisseau et al., 2007; Yang et al., 2007; Solignat et al., 2009; Schwartz and Albert, 2010; Voss et al., 2010; Burt et al., 2011; Kishishita et al., 2013). This system may be useful for research on viral envelope glycoproteins due to the ability of the pseudotype to grow to a high titer in a variety of cell lines. This system can also be used to examine the cell entry properties conferred by the foreign viral proteins. The titer of pseudotype virus obtained with the VSV $\Delta \mathrm{G}^{*}$ system is generally higher than that of the pseudotype retrovirus system (Ogino, et al., 2003). Furthermore, the infection of target cells with VSV pseudotype can be detected as GFPpositive cells by $16 \mathrm{~h}$ post infection because of the high level of GFP expression in the VSV $\Delta \mathrm{G}^{*}$ system (Ogino et al., 2003). Pseudotyping of CHIKV E1E2 protein using the VSV $\Delta \mathrm{G}^{*}$ system may have advantages for studying the functions of CHIKV E1E2 protein as well as for developing a rapid detection system to detect neutralizing antibodies specific for CHIKV infection.

In this study, we developed a VSV pseudotype with CHIKV envelope protein E2E1 (VSV-E2E1) that can be applied as a safe and convenient neutralization assay in order to avoid using wild CHIKV, which should be handled in P3 level laboratory. Because this pseudotyped virus contains a reporter gene, GFP or luciferase, the infection level can be easily detected by measuring the activity of luciferase or the expression of GFP. On the other hand, this pseudotype virus should exhibit the same cell tropism as native CHIKV and will allow the study of the host immune response without the dangers of working with a highly pathogenic virus. In this paper, we evaluated the efficacy of this VSV-E2E1 through neutralization (NT) assay and tested the cell tropism of CHIKV infection.

\section{Materials and Methods}

Cells. The cell lines used in this study were incubated at $37^{\circ} \mathrm{C}$ under a $5 \% \mathrm{CO}_{2}$ atmosphere. Vero (African green monkey kidney epithelial) and HeLa (human cervical adenocarinoma) cells were cultured in Eagle's minimum essential medium (Sigma-Adrich, St. Louis, MO), BEAS-2B cells were cultured in RPMI-1640, 293T cells were cultured in Dulbecco's modified Eagle medium (Life Technologies/Gibco, Foster City, CA). Media were supplemented with $10 \%$ heat-inactivated fetal bovine serum (FBS) (Thermo Fisher Scientific, Waltham, MA) and $100 \mathrm{U} / \mathrm{ml}$ penicillin-100 $\mu \mathrm{g} / \mathrm{ml}$ streptomycin (Life Technologies/Gibco).

Vector construct. The genome from CHIKV strain 37997 (GenBank Acc. No. EU224270) was used for plasmid construction. DNA sequences encoding the structural polyprotein E3-E2-6k-E1 were optimized for expression in human-derived cells (GenScript, Piscataway, NJ; kindly provided by Professor Takeda) (Kishishita et al., 2013) and amplified by PCR using a sense primer containing the start codon and a SacI site (5'-AAAGAGCTCATGAGTCT GGCCCTGCCAGT-3') and an antisense primer containing a stop codon and an NheI site (5'-TTTGCTAGCTCAATGCCT GGAAAATGA-3'). The PCR product was inserted into a pCAGGS. MCSII eukaryotic expression vector (Niwa et al., 1991) to construct pCAGGS-CHIKV E2E1. The expression was confirmed by transfecting 293T cells using Lipofectamine 2000 reagent (Life Technologies/Gibco) according to the manufacturer's protocols and detecting CHIKV E2 proteins by Western blot analysis.

Western blot analysis. The purified virions were analyzed with Western blots. The precipitated virions were solubilized in SDSPAGE sample buffer $(100 \mu \mathrm{l})$ and $20 \mu \mathrm{l}$ aliquots of the samples were applied to $12 \%$ gels. After their transfer onto PVDF membranes (Amersham), viral proteins were immunostained with a monoclonal antibody against CHIKV E2, kindly provided by Professor Desprès (Brehin et al., 2008), for $1 \mathrm{hr}$ at room temperature, followed by horseradish peroxidase-conjugated anti-mouse IgG (Invitrogen), the specific reactions on the membranes were detected with an ECL Western blotting Detection kit (Amersham Pharmacia Biotech).

Preparation of pseudotype virus. At $36 \mathrm{hr}$ after transfection of 293T with pCAGGS-CHIKVE2E1, the 293T cells were infected with VSV $\Delta \mathrm{G}^{*}-\mathrm{G}$ at a multiplicity of infection of 1 for $1 \mathrm{hr}$ at $37^{\circ} \mathrm{C}$. The $293 \mathrm{~T}$ cell monolayer was then washed with $1 \%$ heat-inactivated FCS-PBS three times, and culture medium was added. After $24 \mathrm{hr}$ of incubation at $37^{\circ} \mathrm{C}$ in a $\mathrm{CO}_{2}$ incubator, the culture supernatant was clarified by low-speed centrifugation and partially purified by cen- 
trifugation through $25 \%$ sucrose and stored at $-80^{\circ} \mathrm{C}$. (VSV $\Delta \mathrm{G}^{*}-\mathrm{G}$ was kindly provided by professor Whitt and Matsuura) (Takada $e t$ al., 1997; Matsuura et al., 2001). Pseudotype virus titration was done in Vero E6 cells. Vero E6 cells grown on 96-well plates were infected with $50 \mu \mathrm{l}$ of serially diluted virus stock, after a $1 \mathrm{hr}$ adsorption period, the inoculums were removed, fresh culture medium was added, and the cells were incubated at $37^{\circ} \mathrm{C}$ in a $\mathrm{CO}_{2}$ incubator. At $16 \mathrm{hr}$ post infection, the cells were fixed with $1 \%$ paraformaldehyde in PBS for $10 \mathrm{~min}$ at room temperature, washed with distilled water, and air dried. GFP-expressing cells were counted under a fluorescence microscope (Leica DMIRB). Since VSV pseudotype was unable to produce infectious progeny virus, the number of GFP-positive cells was regarded as infectious units (IU).

Luciferase assay. Luciferase activity in infected cells was determined using the Luciferase Assay Kit (Promega) according to the manufacturer's instructions.

Neutralization assay. A total of $30 \mu \mathrm{l}$ of medium containing 300 IU of VSV-E2E1 pseudotype viruses was incubated with an equal volume of serially diluted antibody against $\mathrm{E} 2$ for $1 \mathrm{hr}$ at $37^{\circ} \mathrm{C}$, then $50 \mu \mathrm{l}$ of the mixture was inoculated onto Vero E6 cell monolayers in 96-well tissue culture plates. After adsorption for $1 \mathrm{hr}$, the mixture was replaced with Eagle minimal essential medium. After a 16-hr incubation period, the cells were collected and prepared for the luciferase activity assay.

Flow Cytometry. Cells infected by the pseudotype viruses were collected by centrifugation, washed in PBS, resuspended in PBS containing $1 \%$ formaldehyde and incubated for $10 \mathrm{~min}$ at room temperature. Cells were washed twice in ice-cold PBS and analyzed on a FACScan flow cytometer (Becton Dickinson Immunocytometry Systems, BDIS). GFP fluorescence was collected after a 530/30$\mathrm{nm}$ bandpass (BP) filter.

\section{Results and Discussion}

\section{Preparation of pseudotype VSV-E2E1 virus}

The region encoding the CHIKV glycoproteins E3, E2, 6k and E1 was optimized for expression in human-derived cells and inserted into an eukaryotic expression vector to construct pCAGGS-CHIKV E2E1. To produce VSV-E2E1 pseudotype viruses, the $293 \mathrm{~T}$ cell lines were infected with $V S V \Delta \mathrm{G}^{*}-\mathrm{G}$ after being transfected with the plasmid pCAGGS-CHIKV E2E1 (Fig. 1a). After $24 \mathrm{hr}$ of incubation at $37^{\circ} \mathrm{C}$, culture supernatants were collected and centrifuged to remove cell debris. VSV-E2E1 pseudotype viruses were partially purified by centrifugation through $25 \%$ sucrose and analyzed by immunoblotting (Fig. 1b). Empty vector pCAGGS was used as the negative control. To determine the infectious titer of the VSV-E2E1 pseudotype virus, cells were infected with the VSVE2E1 pseudotype virus and incubated at $37^{\circ} \mathrm{C}$ for $16 \mathrm{hr}$. The titer of the virus was determined by counting the number of GFP-expressing cells by flow cytometry (data not shown).

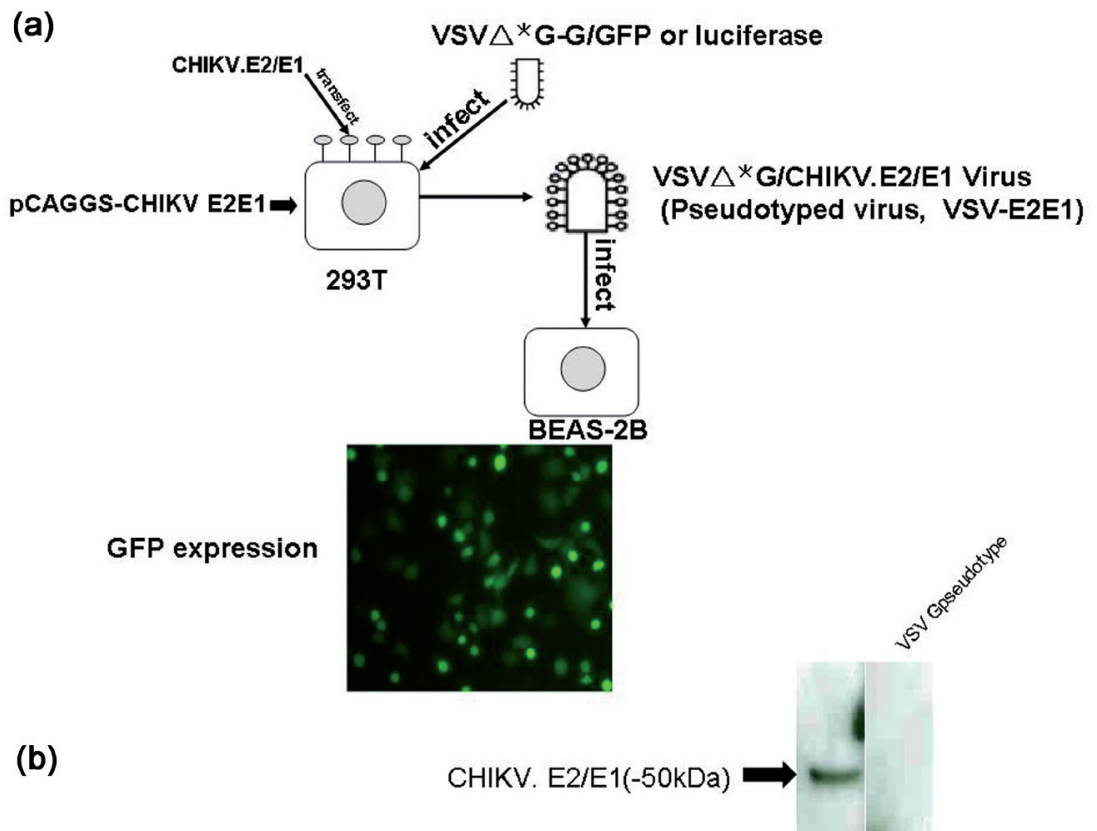

Fig. 1

Generation of pseudotype VSV (a), generation of pseudotype VSVs in 293T cells transiently expressing CHIKV E proteins (b) The E protein expression from VSV-E2E1 pseudotype virus was confirmed by Western blot analysis using monoclonal antibody against E2. The supernatant of $293 \mathrm{~T}$ cells transfected with empty pCAGGS vector served as negative control. 


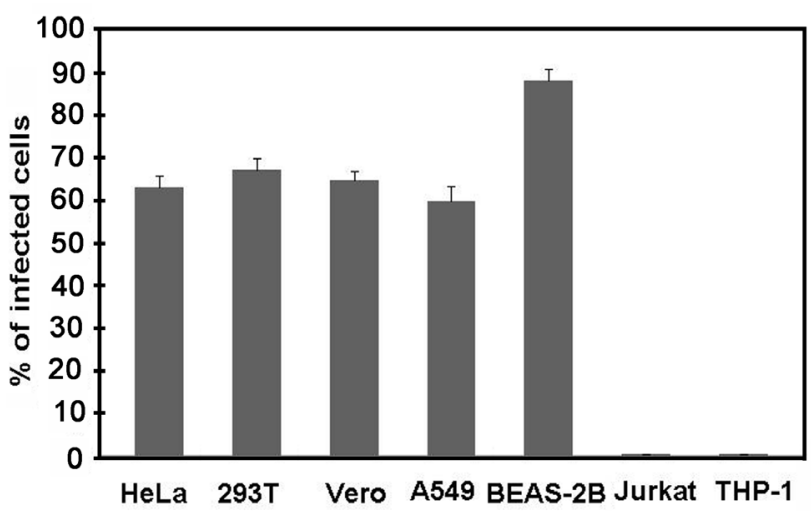

Fig. 2

Cell tropism of CHIKV

Different cell lines were infected with VSV-E2E1 pseudotype virus with GFP as reporter and the percentage of infected cells was assayed. The data are representative of three independent experiments.

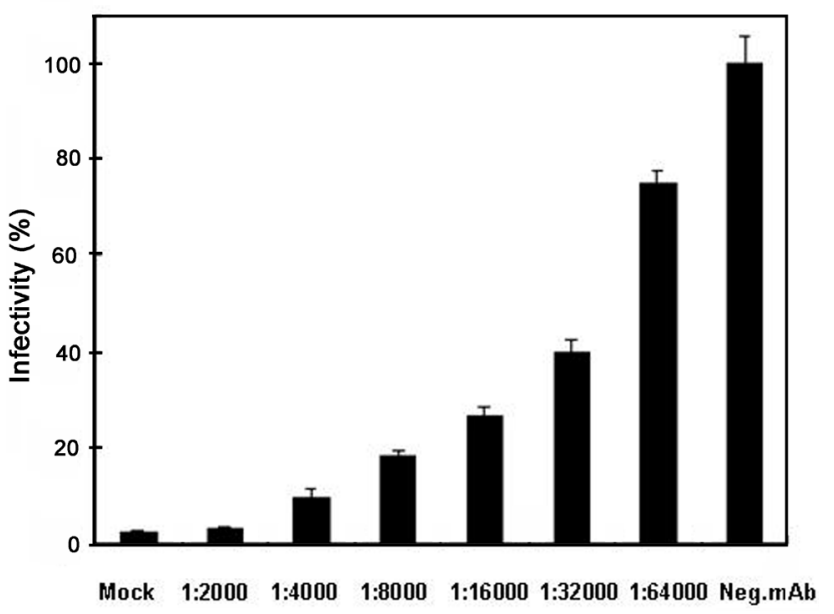

Fig. 3

Neutralization assay using VSV-E2E1 pseudotype virus Serial dilutions of monoclonal antibody to CHIKV E2 were preincubated with VSV-E2E1 pseudotype virus, the mixtures were added to Vero E6 cells in 6-well plate and the luciferase activity was assayed after $16 \mathrm{hr}$. The bar represents a non-neutralized infectivity control, which was set to $100 \%$. The $\%$ infectivity was normalized to the non-neutralized virus infection. Mock: no infection. Normal mouse IgG was used as negative monoclonal antibody.

\section{Cell tropism of VSV-E2E1 pseudotype viruses}

Several human adherent and non-adherent cell lines were used to characterize the tropism of VSV-E2E1 pseudotype virus. Because the VSV-E2E1 pseudotype virus carries the GFP gene as a reporter, the number of cells infected by the VSV-E2E1 pseudotype viruses can be measured by measuring the expression of GFP by using flow cytometry. The adherent cells used in this study were shown to be susceptible to this virus infection. After $16 \mathrm{hr}$ of infection, A549, HeLa, 293T, and BEAS-2B cells were more than $60 \%$ GFP-positive by flow cytometry (Fig. 2). Jurkat (CD4+ T lymphoid cells) and THP-1 were resistant to VSV-E2E1 pseudotype viruses infection.

Neutralization assay using VSV-E2E1 pesudotype luciferase viruses

The VSV-E2E1 pseudotype viruses were incubated with serially diluted antibody against CHIKV E2. The mixture was added to cells, and luciferase activity was measured after 16 hr. Luciferase activity was reduced to the same level as control when the VSV-E2E1 viruses were preabsorbed with lowest dilution (1:2000) of the antibody (Fig. 3). Based on these results we conclude that this system could be conveniently applied to NT assay.

CHIKV characteristics and the widespread distribution of its mosquito vectors make it a potentially highly important emerging pathogen. However, very little is known about the biology of this alphavirus. The envelope protein is the major determinant of alphavirus cell tropism, virus entry and neutralization sensitivity.

In this study, we developed a safe and convenient assay system using a CHIKV VSV-E2E1 pseudotype with a luciferase or GFP reporter, which could be used to characterize CHIKV. This VSV-E2E1 pseudotype virus allows for the investigation of CHIKV-host interactions without the danger of exposure to the highly pathogenic native CHIKV, and it allows experiments to be performed in a biosafety level $2 \mathrm{fa}$ cility. Furthermore, the infectivity of the VSV-E2E1 pseudotype viruses can be examined by a simple fluorescence assay based on the expression of GFP from the pseudotype viral genome or can be measured by the activity of luciferase. The VSV vector is much easier to use than the lentiviral vector because the production of pseudotype lentiviral vectors by the calcium phosphate-mediated method can be technically difficult and tedious (Chen and Okayama, 1987; Yang et al., 2007). The use of the pseudotype virus allows the examination of multiple steps of infection, including both binding to host receptor and the membrane fusion. It might, therefore, be more relevant to examine the native function of CHIKV envelope proteins using this VSV-E2E1 pseudotype virus. As reported by using CHIKV-pseudotyped lentiviral vector, CHIKV infects cells in a $\mathrm{pH}$-dependent manner (Sourisseau et al., 2007). Because this system we used is easier and convenient, it can be applied for the epidemiological studies to screen for the antibody presence in the epidemic area. We have shown that the infectivity of this VSV-E2E1 pseudotype virus has been efficiently neutralized by the antibodies against E2 (Fig. 3).

CHIKVF has a significant public health impact. It was reported that one third of the population on the Reunion 
Island was infected with CHIKV in 2005 and 2006 (Schuffenecker et al., 2006). CHIKV infection is thus a real threat to populations with low herd immunity. CHIKV infection can cause chronic arthralgia, but the mechanism is poorly understood. Detailed examination of CHIKV-infected patients will help to reveal the role of immune responses in the disease development. The presented system could be a helpful tool in such investigations.

Acknowledgements. We would like to thank for Professor Whitt and Matsuura for providing the VSV $\Delta \mathrm{G}^{*}-\mathrm{G}$, Professor Desprès for monoclonal antibody and Professor Takeda for the optimized cDNA of CHIKV E2E1.

\section{References}

Brehin AC, Rubrecht L, Navarro-Sanchez ME, Marechal V, Frenkiel MP, Lapalud P, Laune D, Sall AA, Despres P (2008): Production and characterization of mouse monoclonal antibodies reactive to Chikungunya envelope $\mathrm{E} 2$ glycoprotein. Virology 371, 185-195. http://dx.doi.org/10.1016/j. virol.2007.09.028

Burt FJ, Rolph MS, Rulli NE, Mahalingam S, Heise MT (2011): Chikungunya: a re-emerging virus. Lancet 379, 662-671. http://dx.doi.org/10.1016/S0140-6736(11)60281-X

Chen C, Okayama H (1987): High-efficiency transformation of mammalian cells by plasmid DNA. Mol. Cell. Biol. 7, 2745-2752.

Fukushi S, Mizutani T, Saijo M, Matsuyama S, Miyajima N, Taguchi F, Itamura S, Kurane I, Morikawa S (2005):Vesicular stomatitis virus pseudotyped with severe acute respiratory syndrome coronavirus spike protein. J. Gen. Virol. 86, 2269-2274. http://dx.doi.org/10.1099/vir.0.80955-0

Khan AH, Morita K, Parquet Md Mdel C, Hasebe F, Mathenge EG, Igarashi A (2002): Complete nucleotide sequence of chikungunya virus and evidence for an internal polyadenylation site. J. Gen. Virol. 83, 3075-3084.

Kishishita N, Takeda N, Anuegoonpipat A, Anantapreecha S (2013): Development of a pseudotyped-lentiviralvector-based neutralization assay for chikungunya virus infection. J. Clin. Microbiol. 51, 1389-1395. http://dx.doi. org/10.1128/JCM.03109-12

Matsuura Y, Tani H, Suzuki K, Kimura-Someya T, Suzuki R, Aizaki H, Ishii K, Moriishi K, Robison CS, Whitt MA, Miyamura $\mathrm{T}$ (2001): Characterization of pseudotype VSV possessing HCV envelope proteins. Virology 286, 263-275. http:// dx.doi.org/10.1006/viro.2001.0971

Niwa H, Yamamura K, Miyazaki J (1991): Efficient selection for high-expression transfectants with a novel eukaryotic vector. Gene 108, 193-199. http://dx.doi.org/10.1016/03781119(91)90434-D

Ogino M, Ebihara H, Lee BH, Araki K, Lundkvist A, Kawaoka Y, Yoshimatsu K, Arikawa J (2003): Use of vesicular stoma- titis virus pseudotypes bearing hantaan or seoul virus envelope proteins in a rapid and safe neutralization test. Clin. Diagn. Lab. Immunol. 10, 154-160. http://dx.doi. org/10.1128/cdli.10.1.154-160.2003

Peters C, Dalrymple J (1990): Alphaviruses. In Fields BN, Knipe DM (Eds): Fields Virology. Raven Press, New York, pp. 319-327.

Schuffenecker I, Iteman I, Michault A, Murri S, Frangeul L, Vaney MC, Lavenir R, Pardigon N, Reynes JM, Pettinelli F, Biscornet L, Diancourt L, Michel S, Duquerroy S, Guigon G, Frenkiel MP, Brehin AC, Cubito N, Despres P, Kunst F, Rey FA, Zeller H, Brisse S (2006): Genome microevolution of chikungunya viruses causing the Indian Ocean outbreak. PLoS Med. 3, e263. http://dx.doi.org/10.1371/ journal.pmed.0030263

Schwartz O, Albert ML (2010): Biology and pathogenesis of chikungunya virus. Nat. Rev. Microbiol. 8, 491-500. http:// dx.doi.org/10.1038/nrmicro2368

Solignat M, Gay B, Higgs S, Briant L, Devaux C (2009): Replication cycle of chikungunya: a re-emerging arbovirus. Virology 393, 183-197. http://dx.doi.org/10.1016/j. virol.2009.07.024

Sourisseau M, Schilte C, Casartelli N, Trouillet C, Guivel-Benhassine F, Rudnicka D, Sol-Foulon N, Le Roux K, Prevost MC, Fsihi H, Frenkiel MP, Blanchet F, Afonso PV, Ceccaldi PE, Ozden S, Gessain A, Schuffenecker I, Verhasselt B, Zamborlini A, Saib A, Rey FA, Arenzana-Seisdedos F, Despres P, Michault A, Albert ML, Schwartz O (2007): Characterization of reemerging chikungunya virus. PLoS Pathog 3, e89. http://dx.doi.org/10.1371/journal. ppat.0030089

Strauss EG, Strauss JH (1986): Structure and replication of the alphavirus genome. In Schlesinger M (Ed.): In the Togaviruses and Flaviviruses. Plenum Press, New York, pp. 35-90.

Takada A, Robison C, Goto H, Sanchez A, Murti KG, Whitt MA, Kawaoka Y (1997): A system for functional analysis of Ebola virus glycoprotein. Proc. Natl. Acad. Sci. USA 94, 1476414769. http://dx.doi.org/10.1073/pnas.94.26.14764

Tatsuo H, Okuma K, Tanaka K, Ono N, Minagawa H, Takade A, Matsuura Y, Yanagi Y (2000): Virus entry is a major determinant of cell tropism of Edmonston and wild-type strains of measles virus as revealed by vesicular stomatitis virus pseudotypes bearing their envelope proteins. J. Virol. 74, 4139-4145. http://dx.doi.org/10.1128/ JVI.74.9.4139-4145.2000

Voss JE, Vaney MC, Duquerroy S, Vonrhein C, Girard-Blanc C, Crublet E, Thompson A, Bricogne G, Rey FA (2010): Glycoprotein organization of Chikungunya virus particles revealed by X-ray crystallography. Nature $468,709-712$. http://dx.doi.org/10.1038/nature09555

Yang ZY, Wei CJ, Kong WP, Wu L, Xu L, Smith DF, Nabel GJ (2007): Immunization by avian $\mathrm{H} 5$ influenza hemagglutinin mutants with altered receptor binding specificity. Science 317, 825-828. http://dx.doi.org/10.1126/science.1135165 